\title{
BACKSCATter MATRIX ObSERVATIONS BY THE GV-HSRL
}

\author{
Matthew Hayman, Scott Spuler, Bruce Morley \\ Earth Observing Laboratory, National Center for Atmospheric Research, PO Box 3000, Boulder, CO, 80307, USA, \\ mhayman@ucar.edu
}

\begin{abstract}
The GV-HSRL is a high spectral resolution lidar capable of measuring calibrated backscatter, extinction and circular depolarization from the ground or NSF Gulfstream $\mathrm{V}$ platform. In the spring of 2012, the instrument was modified to measure the full backscatter matrix of atmospheric scatterers. This modification enabled us to investigate the polarization properties of oriented particles and further understand where particles orient and how they may impact depolarization lidar data. Observations were performed from the ground at different times with the lidar's tilt angle at $4^{\circ}, 22^{\circ}$ and $32^{\circ}$ degrees off zenith. This instrument found oriented ice crystals only produced observable polarization effects at the $32^{\circ}$ lidar tilt angle. By contrast, a significant fraction of rain observations have oriented scattering matrices at all three lidar tilt angles. Thus conventional depolarization lidar is generally well suited for characterizing ice crystals but not liquid precipitation.
\end{abstract}

Oriented ice crystals are most commonly investigated by looking for specular scatter from horizontally oriented plates. While this method offers excellent sensitivity to small populations of oriented plats, it has very little capability to determine the fraction of oriented plates if the population is larger than about $1 \%$. We show here that observing $f_{12}$ scattering matrix element at $32^{\circ}$ off zenith may be more effective for estimating the oriented fraction of particles in a volume.

\section{INTRODUCTION}

A typical depolarization lidar system intrinsically assumes that the particles under investigation are randomly oriented, which, by symmetry and reciprocity must have a diagonal backscatter matrix with three degrees of freedom (backscatter, depolarization and where asymmetric particles exist, circular diattenuation) $[1 ; 2]$

$$
\mathbf{F}(\pi)=\beta\left[\begin{array}{cccc}
1 & 0 & 0 & f_{14} \\
0 & 1-d & 0 & 0 \\
0 & 0 & d-1 & 0 \\
f_{14} & 0 & 0 & 2 d-1
\end{array}\right],
$$

where $\beta$ is the volume backscatter coefficient and $d$ is the propensity of the medium to depolarize. The term $f_{14}$ is generally assumed to be zero in atmospheric scattering [3; 4]. The scattering angle argument $\pi$ indicates this is a backscattering phase matrix independent of incident direction.

When this assumption is valid, the standard depolarization ratio is sufficient to fully describe the backscatter properties of the volume. It is known, however, that particles do sometimes orient in the atmosphere. In such cases, the backscatter matrix does not necessarily reduce to the form in Eq. (1) and generally must take the form [3]:

$$
\mathbf{F}\left(\vec{k}_{i},-\vec{k}_{i}\right)=\left[\begin{array}{cccc}
\beta & f_{12} & 0 & f_{14} \\
f_{12} & f_{22} & 0 & 0 \\
0 & 0 & f_{33} & f_{34} \\
f_{14} & 0 & -f_{34} & f_{44}
\end{array}\right] .
$$

where now there are six independent terms in the scattering matrix and the argument $\vec{k}_{i}$ is the incident wave vector, indicating that the scattering matrix is dependent on direction of incidence. The resulting depolarization measurements will depend on several factors, such as the lidar's specific pointing angle, and the polarization transmitted and measured by the lidar. These potential errors are not well quantified due to the very limited number of oriented scattering matrix observations. Thus it is not clear if and when the oriented scattering matrix terms described in Eq. (2) will impact polarization lidar observations.

For a backscatter matrix to appear in the form shown in Eq. (2), three conditions must be met [4]. Oriented particles must be present, the oriented particles must not exhibit symmetry about the incident and backscatter angle and finally the backscatter signal from the oriented particles must be large enough that it is not overwhelmed by the signal from randomly oriented particles. There are very few observations that indicate typical values for oriented scattering matrix terms in the atmosphere. Furthermore there are very few observations to indicate where all three conditions are satisfied for observing these unique scattering matrices.

There has been substantial scientific interest in occurrence of oriented ice crystals $[5 ; 6 ; 7]$. Lidar observations typically take the approach of looking for strong specular reflections from plates by directing the instrument very close to zenith or nadir. This technique has the benefit of being highly sensitive to the presence of even a small population of ice crystals, but comes at the expense of diluting the signatures of all other particles in the volume.

In Hirakata et al. [5], a number of statistics are used to characterize the where 2D (horizontally oriented) and 3D (randomly oriented) plates exist. They notably define a region classified as a "mix" of $2 \mathrm{D}$ and $3 \mathrm{D}$ plates. However, it should be noted that anywhere 2D plates are observed, it is likely 3D plates are also present. Because the backscatter from the 2D plates dominates the signal, 


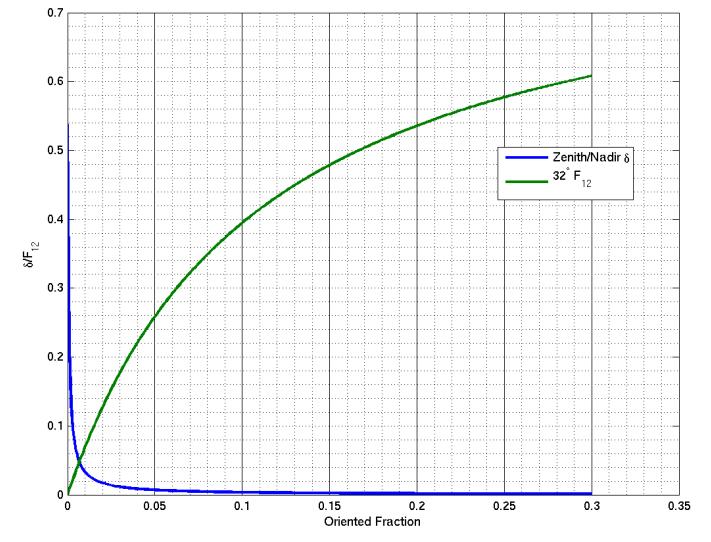

Figure 1: Depolarization ratio (blue) or diattenuation (green) for different oriented fractions.

it is almost impossible to observe the 3D plates or any of their corresponding properties. Furthermore, any attempt to determine the relative mixing of oriented and randomly oriented particles is only practical for very small oriented particle fractions.

Consider a volume consisting of a combination of randomly oriented and oriented plates. The total backscatter is given by the sum of the two backscatter terms

$$
\beta_{T}=\beta_{R}+\beta_{O}
$$

where the subscript $T$ corresponds to the observed total volume, $R$ corresponds to randomly oriented terms and $O$ corresponds to oriented terms. The total depolarization is the backscatter weighted mean of the two backscatter terms

$$
\beta_{T} d_{T}=\beta_{R} d_{R}+\beta_{O} d_{O}
$$

The volume backscatter coefficient is given by the number of particles in the volume multiplied by their backscatter cross section. For the randomly oriented particles this is given by

$$
\beta_{R}=\sigma_{R} n_{R},
$$

and the oriented particles are given by a similar equation, but their backscatter cross section is much larger than that of their randomly oriented counterparts, so let $\sigma_{O}=A \sigma_{R}$ making the oriented volume backscatter coefficient

$$
\beta_{O}=\sigma_{O} n_{O}=A \sigma_{R} n_{O}
$$

The presence of oriented ice crystals are identified by their low depolarization, often inside an ice cloud. The depolarization of a volume consisting of oriented and randomly oriented particles is

$$
d_{T}=\frac{d_{R}+\left(A d_{O}-d_{R}\right) F_{O}}{(A-1) F_{O}+1},
$$
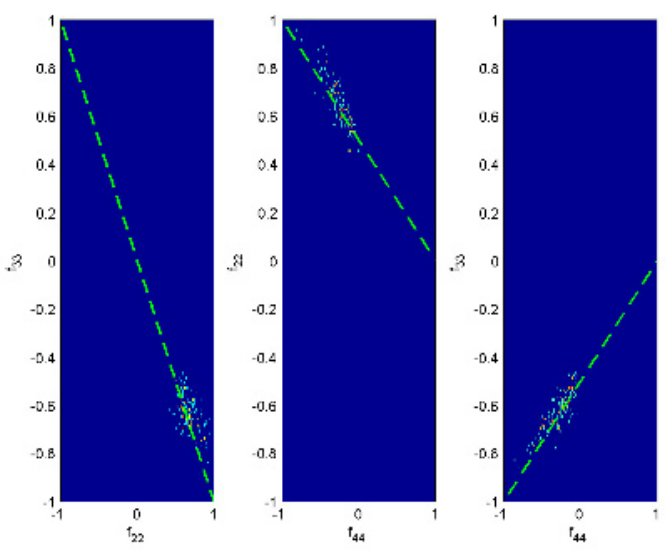

Figure 2: Histograms of the $f_{22}, f_{33}$ and $f_{44}$ of oriented ice crystal observations. The dashed green line shows the expected relationship for randomly oriented ice crystals.

where $d_{T}$ is the total depolarization, $d_{R}$ is the depolarization of the randomly oriented particles, $d_{O}$ is the depolarization of the oriented particles (typically very close to zero), $A$ is the oriented particle enhancement factor and $F_{O}$ is the fraction of oriented particles in the population and is given by

$$
F_{O}=\frac{n_{O}}{n_{O}+n_{R}} .
$$

By the same principles that we obtain Eq. (7) we can also obtain a metric for the total diattenuation of the volume (the $f_{12}$ element of the scattering matrix).

$$
f_{12}^{(T)}=\frac{A f_{12}^{O} F_{O}}{(A-1) F_{O}+1},
$$

Note that $f_{12}=0$ for randomly oriented particles.

A scattering simulation performed by Lei Bi and Ping Yang of $100 \mu \mathrm{m}$ plates has been used to determine that for specular backscatter, $A \approx 1000$. It is assumed that $d_{R}=0.7$ which is near the maximum value we observe with the GV-HSRL, giving maximum sensitivity to oriented fraction. By contrast, for a lidar operating at $32^{\circ}$ off zenith, $A \approx 10$ and $\left|f_{12}\right| \approx 0.75$.

We plot the expected total volume depolarization and diattenuation in Figure 1 as a function of the oriented fraction $F_{O}$. While measuring specular backscatter is highly sensitive to small populations of oriented particles, it is virtually impossible to make distinctions between oriented fractions larger than $1 \%$. By contrast, diattenuation maintains a more consistent sensitivity over a large range of $F_{O}$, making it a more useful parameter for estimating the oriented particle fraction.

\section{OBSERVATIONS}

Observations of scattering matrices presented here are from summer in 2012 and 2013 at lidar tilt angles of $4^{\circ}$, 

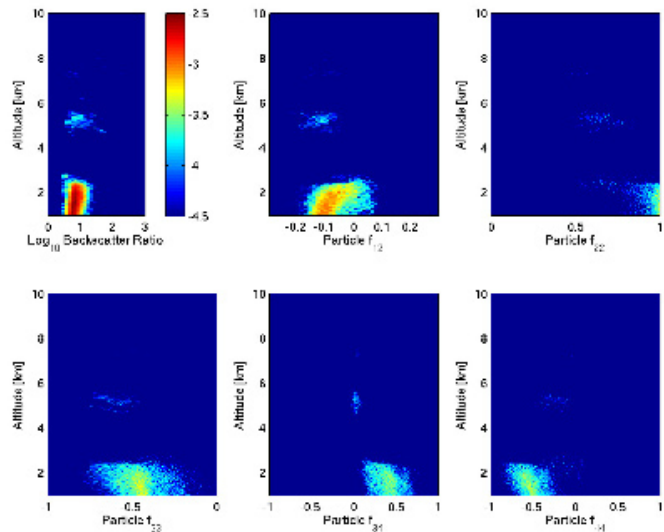

Figure 3: Altitude resolved histograms of scattering matrix elements when observed at a lidar tilt angle of $32^{\circ}$ during June 2012. This figure is originally published in Optics Express in [4] and is used here with permission.

$22^{\circ}$ and $32^{\circ}$ off zenith. All observations were made in Boulder, CO, USA and the lidar technique used for measuring the backscatter matrix is described in [8].

In nearly all cases, oriented ice crystals were only identifiable by significant diattenuation $\left(f_{12}\right)$ terms. In fact, the other matrix terms typically conformed quite well to Eq. (1). Figure 2 shows the relationship between the diagonal terms of oriented ice crystal observations when the lidar was tilted $32^{\circ}$ off zenith. The green dashed line indicates the relationship expected for randomly oriented particles given by

$$
f_{44}=1-2 f_{22}=1+2 f_{33} \text {. }
$$

In [3] the principle parameter used to identify the presence of oriented ice crystals is the orientation parameter $\chi$ which they define as

$$
\chi=\frac{f_{22}+f_{33}}{f_{11}+f_{44}} .
$$

Under the assumption that the single particle backscatter matrix has $f_{33}=f_{44}$ and $f_{22}=1, \chi \rightarrow 1$ as the particle orientation distribution narrows, and $\chi=0$ for a completely randomly oriented population.

The results from the GV-HSRL in Figure 2 conflict with the use of $\chi$ in [3]. In our observations, matrix diagonals do not significantly deviate from the randomly oriented form in Eq. (1) so $\chi$ is always near zero. This may be the result of a significant population of randomly oriented ice crystals also present in the volume. By contrast, [3] uses $\chi$ to identify the presence of oriented ice crystals and finds significant populations with a near zenith directed lidar.

Our processing routines use non-zero off diagonal elements to indicate the presence of oriented particles in a
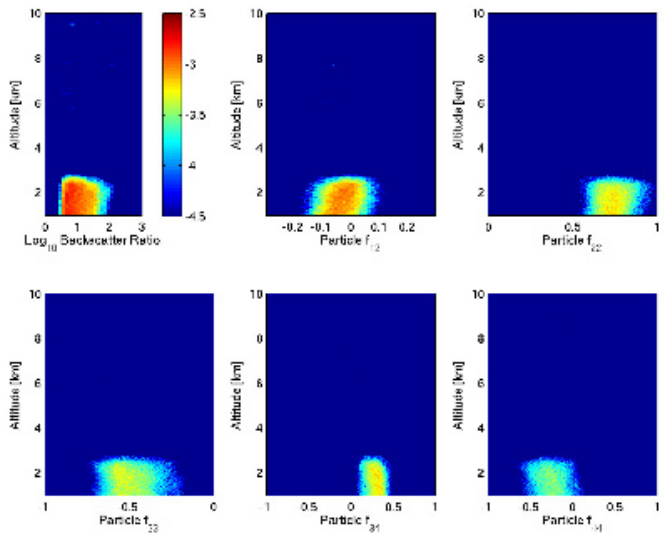

Figure 4: Altitude resolved histograms of scattering matrix elements when observed at a lidar tilt angle of $22^{\circ}$ during July 2012. This figure is originally published in Optics Express in [4] and is used here with permission.

volume. In particular, we look for non-zero diattenuation $\left(f_{12}\right)$ and retardance $\left(f_{34}\right)$. The altitude resolved histograms of all oriented scattering matrix elements are shown in Figures 3, 4 and 5 for lidar tilt angles of $32^{\circ}$, $22^{\circ}$ and $4^{\circ}$ respectively. These data sets were recorded in the summer and observations of oriented particles above $3 \mathrm{~km}$ are generally regarded as oriented ice crystals where observations below $3 \mathrm{~km}$ are rain.
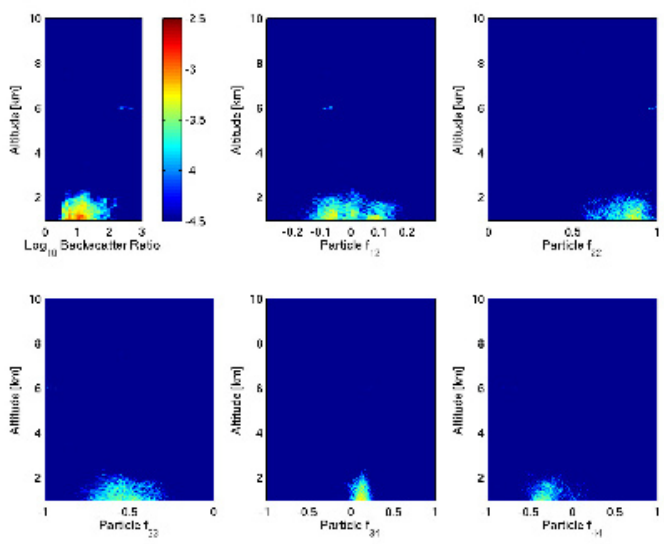

Figure 5: Altitude resolved histograms of scattering matrix elements when observed at a lidar tilt angle of $4^{\circ}$ during August 2013. This figure is originally published in Optics Express in [4] and is used here with permission.

The oriented ice observations only appear when the lidar is tilted $32^{\circ}$ off zenith. In the other two cases, it appears that either the off diagonal terms are small and/or the oriented backscatter signal is overwhelmed by randomly oriented particles. Even at $32^{\circ}$ tilt angle, only the $f_{12}$ term shows significant contribution from oriented parti- 
cles. Noting that the maximum measured $\left|f_{12}\right|$ is approximately 0.2 we can use Figure 1 to roughly estimate that the oriented fraction of particles are typically between 0 and $3.5 \%$.

In contrast to ice observations, the rain scattering matrices appears to contain significant oriented scattering terms at all three lidar tilt angles. Rain scattering matrices exhibit significant diattenuation $\left(f_{12}\right)$ and retardance $\left(f_{34}\right)$ and the diagonal terms generally do not conform to Eq. (10).

\section{CONCLUSION}

Observations performed by the GV-HSRL of oriented scattering matrices showed that oriented polarization effects are quite rare in ice clouds. Their peak occurrence was found to be of the order of $1 \%$ at altitudes of $5 \mathrm{~km}$ and they were only observed in significant numbers when the lidar was tilted at $32^{\circ}$ off zenith. Even when oriented particle polarization effects were observed, they were only significant in the $f_{12}$ parameter and diagonal terms seemed to conform relatively well to the functional form of randomly oriented particles. It is notable that these observations differ significantly from [3] where near zenith pointing lidar observed and characterized oriented ice crystals using deviations from the expected diagonal relationship of randomly oriented particles.

Our observations also show that rain has significant oriented scattering terms and cannot be reliably described by the linear depolarization ratio. Also, when circular depolarization is reported for rain, the angle of incidence should be specified. It may be possible to improve characterization of liquid precipitation using scattering matrix observations of rain. In a number of profiles, we see size sorting common to rain storms, where rain is highly oriented at the beginning and becomes less so as the storm passes.

\section{ACKNOWLEDGMENTS}

The National Center for Atmospheric Research is sponsored by the National Science Foundation.

\section{REFERENCES}

1. Flynn, C. J., Mendoza, A., Zheng, Y., Mathur, S. 2007: Novel polarization-sensitive micropulse lidar measurement technique. Opt. Express 15, pp. 2785 2790.

2. Gimmestad, G. 2008: Re-examination of depolarization in lidar measurements. Appl. Opt. 47, pp. 3795 3802.

3. Kaul, B. V., Samokhvalov, I. V., Volkov, S. N. 2004: Investigating particle orientation in cirrus clouds by measuring backscattering phase matrices with lidar. Appl. Opt., 43, pp. 6620-6628.

4. Hayman, M., Spuler, S., Morley, B. 2014: Polarization lidar observations of backscatter phase matrices from oriented ice crystals and rain. Opt. Express, 22, pp. 16976-16990.
5. Hirakata, M., Okamoto, H., Hagihara, Y., Hayasaka, T., Oki, R. 2014: Comparison of global and seasonal characteristics of cloud phase and horizontal ice plates derived from CALIPSO with MODIS and ECMWF. J. Atmos. Oceanic Technol., 31, pp. 21142130 .

6. Noel. V. and Sassen K. 2005: Study of planar ice crystal orientations in ice clouds from scanning polarization lidar observations. J. Appl. Meteor., 44, pp. 653-664.

7. Wesbrook, C. D. 2008: The fall speeds of sub$100 \mu \mathrm{m}$ ice crystals. Q. J. R. Meteorol. Soc., 134, pp. 1243-1251.

8. Hayman, M., Spuler, S., Morley, B., VanAndel, J. 2012: Polarization lidar operation for measuring backscatter phase matrices of oriented scatterers. Opt. Express, 20, pp. 29553-29567. 